\title{
A NEW SOLUTION OF THE EINSTEIN-MAXWELL EQUATIONS FOR A SYSTEM WITH MASS, MAGNETIC MOMENT, CHARGE, AND ANGULAR MOMENTUM*
}

\author{
LOUIS WITTEN \\ University of Cincinnati, Cincinnati, Ohio, U.S.A.
}

\begin{abstract}
A five parameter solution of the combined Einstein-Maxwell equations is given which describes a source containing mass, electric charge, magnetic dipole, higher multipole moments of all three kinds, and angular momentum. The solution is asymptotically flat and has a singular infinite red shift surface. Possible relevance of the solution to black hole physics is discussed.
\end{abstract}

* Based on a paper entitled 'A Five Parameter Exterior Solution of the Einstein-Maxwell Field Equations' by F. Paul Esposito and Louis Witten, Phys. Rev. D8, 3302 (1974). 